hep-th/0508174

\title{
Exact Black Hole Degeneracies and the Topological String
}

\author{
David Shih ${ }^{a}$ and Xi Yin ${ }^{b}$ \\ ${ }^{a}$ Department of Physics, Princeton University, Princeton, NJ 08544, USA \\ ${ }^{b}$ Jefferson Physical Laboratory, Harvard University, Cambridge, MA 02138, USA
}

\begin{abstract}
Motivated by the recent conjecture of Ooguri, Strominger and Vafa, we compute the semi-canonical partition function of BPS black holes in $\mathcal{N}=4$ and $\mathcal{N}=8$ string theories, to all orders in perturbation theory. Not only are the black hole partition functions surprisingly simple; they capture the full topological string amplitudes, as expected from the OSV conjecture. The agreement is not perfect, however, as there are differences between the black hole and topological string partition functions even at the perturbative level. We propose a minimal modification of the OSV conjecture, in which these differences are understood as a nontrivial measure factor for the topological string.
\end{abstract}

August, 2005 


\section{Contents}

1. Introduction . . . . . . . . . . . . . . . . . . . . . . . . . . . . . . . . 1

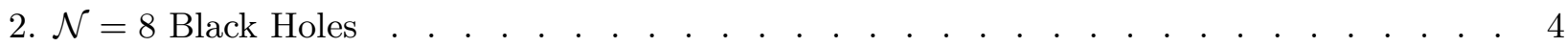

2.1. Preliminaries ............................... . 4

2.2. The full OSV transform . . . . . . . . . . . . . . . . . . . . . . . . . . 6

2.3. Rewriting in terms of the topological string on $T^{6}$. . . . . . . . . . . . . . . . 7

2.4. The general OSV transform . . . . . . . . . . . . . . . . . . . . . . . . 8

3. $\mathcal{N}=4$ Black Holes . . . . . . . . . . . . . . . . . . . . . . . . . . . . . . . 9

3.1. Preliminaries . . . . . . . . . . . . . . . . . . . . . . . . . 9

3.2. The full OSV transform . . . . . . . . . . . . . . . . . . . . . . . . . 10

3.3. Rewriting in terms of the topological string on $K 3 \times T^{2}$. . . . . . . . . . . . . . 12

3.4. The general OSV transform . . . . . . . . . . . . . . . . . . . . . . . . . 13

4. Summary and Discussion . . . . . . . . . . . . . . . . . . . . . . . . . . . . 13

4.1. Summary of results . . . . . . . . . . . . . . . . . . . . . . . . . . . . . 13

4.2. The holomorphic anomaly and the metric on moduli space . . . . . . . . . . . . 14

4.3. D-instantons . . . . . . . . . . . . . . . . . . . . . . . . . . . . . . . . 17

4.4. Non-perturbative corrections . . . . . . . . . . . . . . . . . . . . . . . . . 18

Appendix A. Nonperturbative Corrections to the $\mathcal{N}=4$ OSV Transform . . . . . . . . 18

\section{Introduction}

A simple relation between the partition function of BPS black holes and that of topological strings, of the form

$$
Z_{B H}(p, \phi)=\sum_{q} \Omega_{B H}(p, q) e^{-q \cdot \phi}=\left|Z_{t o p}(p+i \phi)\right|^{2}
$$

was recently proposed by Ooguri, Strominger and Vafa (OSV) in [1]. Subsequently, much work has been done to clarify and test this proposal [2-15]. Clarification is definitely needed, as a quick glance at (1.1) shows that the original proposal of OSV is schematic at best - taking it literally immediately leads to problems. For instance, the LHS of (1.1) is manifestly invariant under integer shifts of $\phi, \phi \rightarrow \phi+2 \pi i k$, while the RHS is generally not. Another point that needs clarification is the definition of black hole partition function $\Omega_{B H}(p, q)$ : in general, there are multiple "supersymmetric indices" which have the correct asymptotics and could potentially be used in (1.1).

Since the precise formulation of the OSV proposal is currently lacking, it is especially important to have examples of black holes whose degeneracies are (a) known exactly, and (b) sufficiently simple that the OSV transform $Z_{B H}(p, \phi)$ can be evaluated explicitly. 
The OSV conjecture was originally formulated for BPS black holes in $\mathcal{N}=2$ compactifications of string theories. The classical entropy (and certain subleading corrections) of such black holes has been understood microscopically [16.,17]. However, general formulas for the exact degeneracies of large $\mathcal{N}=2$ black holes are not known $\mathbb{1}$ The only large $\mathcal{N}=2$ black holes whose exact degeneracies are known are those derived from "compactifications" on certain local Calabi-Yau 3-folds. Recent progress along this direction includes [2, 田.

The difficulty of deducing exact degeneracies for black holes in $\mathcal{N}=2$ compactifications stems from the complexity of the underlying Calabi-Yau. This motivates the study of compactifications and black holes with more supersymmetry, which typically involve simpler Calabi-Yaus. The two examples which we will study in this paper are type IIA string theory compactified on $K 3 \times T^{2}$, which has $\mathcal{N}=4$ supersymmetry in four dimensions; and type IIA compactified on $T^{6}$, which has $\mathcal{N}=8$ supersymmetry in four dimensions.

The most well-studied black holes in this context are the small 1/2 BPS black holes in $\mathcal{N}=4$ compactification on $K 3 \times T^{2}$ [3,8, 15]. These are known as Dabholkar-Harvey states [18,19], because they are dual to wound fundamental strings in the dual heterotic description. Their exact degeneracies are quite simple, being given by the partition function of the heterotic string. Unfortunately, since their classical entropy vanishes, it is not entirely clear whether the OSV proposal should apply to these black holes.

In order to have nonvanishing classical entropy, BPS black holes in $\mathcal{N}=4$ and $\mathcal{N}=8$ string theories must preserve exactly $1 / 4$ and $1 / 8$ of the supersymmetries, respectively. Formulas for the exact degeneracies of such black holes were recently derived in [20] and [21]. (The formula for the exact degeneracies of $1 / 4$ BPS black holes was conjectured by Dijkgraaf, Verlinde and Verlinde nearly 10 years ago in [22].)

These exact degeneracies have all been tested against the topological string amplitudes in some way or another [3, 8, [15, 6, [14]. In all these tests, however, the approach has been to either Legendre or Laplace transform the topological string amplitudes and compare them with the black hole entropy. Only partial agreement has been found this way, in part because of various ambiguities, e.g. in the contour and measure of integration, inherent in this approach.

In this paper we will adopt a complementary viewpoint - we will directly compare the semi-canonical black hole partition function in $\mathcal{N}=4$ and $\mathcal{N}=8$ string theories with

1 The exact degeneracy of certain classes of small $\mathcal{N}=2$ black holes is known (see e.g. [15]). By large (small), we mean black holes with nonvanishing (vanishing) classical horizon area. 
the topological string partition function. This approach has several advantages. Since it involves a sum over charges instead of an integral over potentials, it avoids the ambiguities regarding the contour and measure of integration. Moreover, the direct approach can, in principle, provide a non-perturbative completion of the topological string amplitudes.

Regardless of how one performs the comparison with the topological string, there is always one ambiguity of the OSV conjecture in the context of $\mathcal{N}=4$ and $\mathcal{N}=8$ string theories. This comes from the extra charges associated with the KK gauge fields. These charges are not present in $\mathcal{N}=2$ compactification.2 Since the original proposal of OSV was in the context of $\mathcal{N}=2$ compactification, it is unclear whether we should sum over these extra charges when computing the semi-canonical partition function (1.1). On the other hand, there is good reason to suppose that the attractor mechanism applies just as well to these extra charges (see e.g. 23.).

Perhaps the most straightforward prescription is to turn off these extra charges and compare the "reduced" OSV transform of $\Omega(p, q)$ with a similarly reduced topological string partition function. The other natural prescription is to perform a "full" OSV transform of the exact degeneracies with respect to all the charges. To parameterize our ignorance, we will introduce an integer $n$ and consider the most general OSV transform

$$
Z_{B H}^{(n)}(p, \phi) \equiv \sum_{q_{1}, \ldots, q_{n}} \sum_{q_{a}} \Omega_{B H}(p, q) e^{-q \cdot \phi}
$$

where $q_{a}$ denote the charges in $\mathcal{N}=2$ graviton and vector multiplets, and $q_{1}, \ldots, q_{n}$ denote the extra charges associated with the $\mathcal{N}=2$ gravitini multiplets. Here $\Omega_{B H}(p, q)$ is the proper supersymmetric index of the black holes as defined in [24,20,21]. Our goal for the rest of this paper will be to calculate, and then to interpret (1.2).

For various technical reasons, we will restrict ourselves to vanishing D6 brane charge; other than this restriction, our results will be valid for arbitrary charge configurations.

Interestingly, we will see that the answer for any $n$ can be expressed in terms of the topological string amplitude in simple ways. In particular, for the "full" OSV transform, we find

$$
Z_{B H}^{(f u l l)}(p, \phi)=\sum_{\phi \rightarrow \phi+2 \pi i k}\left|Z_{\text {top }}\right|^{2}\left|g_{t o p}\right|^{2\left(b_{1}-2\right)} V_{X}+\mathcal{O}\left(e^{-V_{X} / g_{\text {top }}^{2}}\right)
$$

2 One way to see this is to note that in an $\mathcal{N}=2$ reduction of the full supersymmetry, these gauge fields belong to $\mathcal{N}=2$ gravitini multiplets, which do not arise in $\mathcal{N}=2$ compactification. 
where $V_{X}$ is the volume of the Calabi-Yau $X=K 3 \times T^{2}$ or $T^{6}$, and $b_{1}$ is the first Betti number of $X$. Additionally, we find that in the $\mathcal{N}=8$ case, the nonperturbative corrections can be summed up completely, yielding a closed-form expression for the exact OSV transform.

With a bit of guesswork, we also propose the following unified presentation of the most general OSV transform:

$$
Z_{B H}^{(n)}(p, \phi)=\sum_{\phi \rightarrow \phi+2 \pi i k}\left|Z_{\text {top }}^{\prime}\right|^{2} \sqrt{\operatorname{det} g^{(q)}}+\text { non pert }
$$

where $\left|Z_{\text {top }}^{\prime}\right|^{2}$ is the square of the topological string partition function including the holomorphic anomaly, and $g^{(q)}$ is a "quantum-corrected" metric on the moduli space of the topological string on $X$ (to be defined below).

This paper is organized as follows. In section 2 we study the $1 / 8$ BPS black holes in $\mathcal{N}=8$ string theory; compute the semi-canonical partition function $Z_{B H}(p, \phi)$ including the nonperturbative corrections; and compare to the topological string. In section 3 we shall study the analogous problem for $1 / 4$ BPS black holes in $\mathcal{N}=4$ string theory, although the analysis of the nonperturbative corrections will be left to appendix A. Finally, section 4 summarizes our results and discusses the possible implications.

\section{2. $\mathcal{N}=8$ Black Holes}

\subsection{Preliminaries}

In this section, we wish to consider the OSV transform of the exact degeneracies of $1 / 8$ BPS $\mathcal{N}=8$ black holes. These black holes can be realized as wrapped branes and strings in a type IIA compactification on $T^{6}$. A formula for their exact degeneracies was derived recently in [21]. It takes the form

$$
\Omega_{\mathcal{N}=8}(p, q) \equiv \sum_{J^{3}, B P S \text { states }} 2\left(J^{3}\right)^{2}(-)^{2 J^{3}}=d(J(p, q))=\oint d \rho F(\rho) e^{-2 \pi i \rho J(p, q)}
$$

where the two ingredients of the RHS are:

1. $F(\rho)$, a modular form

$$
F(\rho)=\frac{\theta_{3}(2 \rho)}{\eta(4 \rho)^{6}}=\frac{\eta(2 \rho)^{5}}{\eta(4 \rho)^{8} \eta(\rho)^{2}} \sim C \rho^{5 / 2} e^{\pi i / 8 \rho}(1+\ldots)
$$


The $\ldots$ represents an expansion in powers of $e^{-\pi i / 2 \rho}$. These corrections contribute order $\mathcal{O}\left(e^{-Q^{2}}\right)$ terms to $\Omega_{\mathcal{N}=8}$, which are expected to be non-perturbative from the topological string point of view.

2. $J(p, q)$, the unique quartic invariant of the $\mathcal{N}=8 \mathrm{U}$-duality group, known as the Cremmer-Julia invariant [25]. In a suitable basis (see e.g. [26,22]), this invariant takes the form

$$
J=-\operatorname{Tr}(Y Z Y Z)+\frac{1}{4}(\operatorname{Tr} Y Z)^{2}-4(\operatorname{Pf} Y+\operatorname{Pf} Z)
$$

where $Y$ and $Z$ are $8 \times 8$ antisymmetric real matrices which encode the charges of wrapped branes and strings.

From the $\mathcal{N}=8$ point of view $Y$ and $Z$ are naturally electric and magnetic charges. However, in performing the OSV transform, we should assign the magnetic and electric charges that are consistent with the coupling of $\mathcal{N}=2$ vector, graviton and gravitini multiplets, as follows:

$$
Y=\left(\begin{array}{ccc}
P & -p^{1} & -p^{2} \\
p^{1 T} & 0 & -q_{0} \\
p^{2 T} & q_{0} & 0
\end{array}\right), \quad Z=\left(\begin{array}{ccc}
Q & -q_{1} & -q_{2} \\
q_{1}^{T} & 0 & -p^{0} \\
q_{2}^{T} & p^{0} & 0
\end{array}\right)
$$

Here $P$ and $Q$ are $6 \times 6$ antisymmetric matrices, and $q_{1}, q_{2}, p^{1}, p^{2}$ are six-dimensional vectors. These are divided into electric charges $q_{\Lambda}$ and magnetic charges $p^{\Lambda}, \Lambda=0, \ldots, 27$, as follows:

$$
q_{\Lambda}=\left\{q_{0}, Q_{i j}, q_{1 i}, q_{2 i}\right\}, \quad p^{\Lambda}=\left\{p^{0}, P^{i j}, p^{1 i}, p^{2 i}\right\}
$$

Physically, $P^{i j}$ and $Q_{i j}$ are identified with $T^{6}$-wrapped D4 and D2 brane charges respectively, with $i, j=1, \ldots, 6$ representing the 1 -cycles of the $T^{6}$. Meanwhile, $q_{1 i}$ and $q_{2 i}$ correspond to KK monopole and NS5 brane charges, respectively, while $p^{1 i}$ and $p^{2 i}$ correspond to KK momentum and F-string winding charges, respectively. Finally, $q_{0}$ is D0 brane charge, while $p^{0}$ is D6 brane charge.

As mentioned in the introduction, we will focus exclusively on the case $p^{0}=0$ in this paper. In this limit, one can check that (2.3) simplifies to

$$
J=4(p)^{3}\left(q_{0}+\frac{1}{12} D^{A B} q_{A} q_{B}\right)
$$

where $A, B=1, \ldots, 27$ are the collective indices for the 27 electric and magnetic charges other than $q_{0}$ or $p^{0}$, and we have defined

$$
(p)^{3}=D_{A B C} p^{A} p^{B} p^{C}=\operatorname{Pf} P+p^{1 T} P p^{2}, \quad D_{A B}=D_{A B C} p^{C}, \quad D^{A B} D_{B C}=\delta_{C}^{A} .
$$


This result illustrates the advantages of assuming $p^{0}=0$, in the following way. Notice how $J \sim q^{2}$ for $p^{0}=0$. On the other hand, for $p^{0} \neq 0$, one can show that $J \sim p^{0} q^{3}$ for large electric charges $q$. Since the black hole degeneracy grows like $\Omega_{B H}(p, q) \sim \exp (\pi \sqrt{J(p, q)})$ in the large charge limit, convergence of the OSV transform (1.2) seems problematic for $p^{0} \neq 0$, while it is less so for $p^{0}=0$. Thus, in addition to simplifying the calculations considerably, the restriction $p^{0}=0$ also improves the convergence of the OSV transform (1.2).

Now that we have described the various components of the exact degeneracy formula (2.1), let us turn to evaluating the OSV transform

$$
Z_{\mathcal{N}=8}(p, \phi)=\sum_{q_{0}, q_{A}} \Omega_{\mathcal{N}=8}(p, q) e^{-q_{0} \phi^{0}-q_{A} \phi^{A}}
$$

\subsection{The full $O S V$ transform}

Let us start by rewriting the black hole partition sum

$$
\begin{aligned}
Z_{\mathcal{N}=8}(p, \phi) & =\sum_{q_{0}, q_{A}} \Omega_{\mathcal{N}=8}(p, q) e^{-q_{0} \phi^{0}-q_{A} \phi^{A}} \\
& =\frac{1}{(p)^{3}} \sum_{\phi^{0} \rightarrow \phi^{0}+2 \pi i k^{0}} \sum_{q_{A}} \sum_{J} d(J) \exp \left[-\phi^{0}\left(\frac{J}{4(p)^{3}}-\frac{1}{12} D^{A B} q_{A} q_{B}\right)-q_{A} \phi^{A}\right]
\end{aligned}
$$

where we used (2.6) to express $q_{0}$ in terms of $J$, and the sum over $k^{0}$ is in the range $0 \leq k^{0} \leq 4(p)^{3}-1$. The sum over $J$ gives precisely the modular form $F\left(i \phi^{0} / 8 \pi(p)^{3}\right)$ defined in (2.2). Meanwhile, we can evaluate the sum over $q_{A}$ via Poisson resummation. Then we are left with

$$
Z_{\mathcal{N}=8}(p, \phi)=\sum_{\phi^{0} \rightarrow \phi^{0}+2 \pi i k^{0}} \sum_{\phi^{A} \rightarrow \phi^{A}+2 \pi i k^{A}}\left(\phi^{0}\right)^{-\frac{27}{2}}(p)^{\frac{21}{2}} F\left(\frac{i \phi^{0}}{8 \pi(p)^{3}}\right) \exp \left(-\frac{3 D_{A B C} \phi^{A} \phi^{B} p^{C}}{\phi^{0}}\right)
$$

Finally, we can make use of the modular property of $F(\rho)$,

$$
F(\rho)=\rho^{5 / 2} e^{\frac{\pi i}{8 \rho}} \tilde{F}(-1 / 4 \rho)
$$

3 Due to the non-positive-definiteness of $D_{A B}$, the sum over $q_{A}$ is, strictly speaking, not convergent. We will regularize the divergence by performing a formal Poisson resummation. A more careful regularization was considered in section 6 of [15]. In any event, we expect our results to be regularization independent.

4 Here and throughout we will neglect overall numerical factors. 
with

$$
\tilde{F}(\rho)=e^{\pi i \rho / 2} \frac{\theta_{3}(2 \rho)}{\eta(\rho)^{6}}=1+\mathcal{O}\left(e^{2 \pi i \rho}\right)
$$

and write $(2.10)$ as

$$
Z_{\mathcal{N}=8}(p, \phi)=\sum_{\phi \rightarrow \phi+2 \pi i k}\left(\phi^{0}\right)^{-11}(p)^{3} \tilde{F}\left(\frac{2 \pi i(p)^{3}}{\phi^{0}}\right) \exp \left(\frac{\pi^{2}(p)^{3}}{\phi^{0}}-\frac{3 D_{A B C} \phi^{A} \phi^{B} p^{C}}{\phi^{0}}\right)
$$

In the next subsection, we will see how this fairly simple expression becomes even simpler when it is recast in terms of the topological string on $T^{6}$.

\subsection{Rewriting in terms of the topological string on $T^{6}$}

The topological string on $T^{6}$ is essentially trivial. The prepotential consists of only a tree-level term, determined by the intersection form on $T^{6}$ :

$$
Z_{\text {top }}=e^{F_{\text {top }}}, \quad F_{\text {top }}=\frac{(2 \pi i)^{3} D_{A B C} t^{A} t^{B} t^{C}}{g_{\text {top }}^{2}}
$$

Here $t^{A}$ are the Kähler moduli of $T^{6}$. Together with the topological string coupling $g_{t o p}$, they are related to the magnetic charges and electric potentials defined above via the attractor equations:

$$
t^{A}=\frac{X^{A}}{X^{0}}=\frac{p^{A}+i \phi^{A} / \pi}{p^{0}+i \phi^{0} / \pi}, \quad g_{\text {top }}=\frac{4 \pi i}{X^{0}}=\frac{4 \pi i}{p^{0}+i \phi^{0} / \pi}
$$

Keep in mind that we are considering only the case $p^{0}=0$ in this work. Notice also that we have slightly generalized the notion of $F_{\text {top }}$ to include the 12 moduli in the $\mathcal{N}=2$ gravitini multiplets, $t^{1 i}$ and $t^{2 i}$. This is well-motivated from U-duality, and we will see that it leads the correct answer.

Another quantity of interest is the Kähler potential of the underlying special geometry (the factor of $1 / \pi$ is for later convenience):

$$
e^{-K}=\frac{1}{\pi} \sum_{\Lambda=0}^{27} \operatorname{Re} \bar{X}^{\Lambda} \partial_{\Lambda} F_{t o p}
$$

For $p^{0}=0$, this is simply

$$
e^{-K}=\frac{2 \pi(p)^{3}}{\phi^{0}}
$$


Now we are ready to recast the OSV transform in terms of the topological string on $T^{6}$. Using (2.14)-(2.17), we see that (2.13) can be written as

$$
Z_{\mathcal{N}=8}=\sum_{\phi \rightarrow \phi+2 \pi i k}\left|Z_{\text {top }}\right|^{2}\left|g_{\text {top }}\right|^{10} e^{-K} \tilde{F}\left(i e^{-K}\right)
$$

We stress that (2.18) is only derived in the case $p^{0}=0$. We also note that the sum over $k^{0}$ is restricted to the range $0 \leq k^{0}<4(p)^{3}$, although $(p)^{3}$ is large in the limit of small $g_{\text {top }}$ while fixing the size of the $T^{6}$.

To highlight the $g_{t o p}$ dependence, it is useful to rewrite (2.18) in terms of the volume of the $T^{6}$ :

$$
V_{T^{6}}=\left|g_{t o p}\right|^{2} e^{-K}
$$

Then (2.18) can be rewritten as

$$
Z_{\mathcal{N}=8}=\sum_{\phi \rightarrow \phi+2 \pi i k}\left|Z_{\text {top }}\right|^{2}\left|g_{\text {top }}\right|^{8} V_{T^{6}} \tilde{F}\left(\frac{i V_{T^{6}}}{\left|g_{\text {top }}\right|^{2}}\right)
$$

¿From (2.12), we see that this is essentially $\left|Z_{\text {top }}\right|^{2}$ up to corrections that are nonperturbative in $g_{t o p}$,

$$
Z_{\mathcal{N}=8}=\sum_{\phi \rightarrow \phi+2 \pi i k}\left|Z_{\text {top }}\right|^{2}\left|g_{t o p}\right|^{8} V_{T^{6}}+\text { nonpert }
$$

Interestingly, the nonperturbative corrections go like $\sim e^{-2 \pi V_{T^{6}} /\left|g_{t o p}\right|^{2}}$. We will discuss possible interpretations of this in section 4 .

\subsection{The general OSV transform}

Before we go on to the case of $1 / 4 \mathrm{BPS} \mathcal{N}=4$ black holes, let us take a moment to consider a "reduced" OSV transform which is natural from the point of view of $\mathcal{N}=2$ supersymmetry. Here we transform $Z_{\mathcal{N}=8}(p, q)$ only with respect to the the charges $q_{0}$ and $Q_{i j}(i, j=1, \cdots, 6)$ which are associated to $\mathcal{N}=2$ vector multiplets. The extra magnetic charges $p^{1}, p^{2}$ and electric potentials $\phi^{1}, \phi^{2}$ in $\mathcal{N}=2$ gravitini multiplets are turned off. The calculation of this "reduced" $\mathrm{BH}$ partition function is almost identical to the previous section, and the result is

$$
\begin{aligned}
Z_{\mathcal{N}=8}^{r e d} & =\sum_{\phi \rightarrow \phi+2 \pi i k}\left|Z_{\text {top }}\right|^{2}\left|g_{\text {top }}\right|^{6} e^{+K} \tilde{F}\left(i e^{-K}\right) \\
& =\sum_{\phi \rightarrow \phi+2 \pi i k}\left|Z_{t o p}\right|^{2}\left|g_{t o p}\right|^{8} \frac{1}{V_{T^{6}}}+\text { nonpert }
\end{aligned}
$$


For the sake of completeness, let us also compute the most general "reduced" partition function, with $0 \leq n \leq 12$ of the gravitini charges summed up:

$$
\begin{aligned}
Z_{\mathcal{N}=8}^{(n)} & =\sum_{\phi \rightarrow \phi+2 \pi i k}\left|Z_{\text {top }}\right|^{2}\left|g_{\text {top }}\right|^{8}\left(V_{T^{6}}\right)^{n / 6-1} \tilde{F}\left(i e^{-K}\right) \\
& =\sum_{\phi \rightarrow \phi+2 \pi i k}\left|Z_{\text {top }}\right|^{2}\left|g_{\text {top }}\right|^{8}\left(V_{T^{6}}\right)^{n / 6-1}+\text { nonpert } .
\end{aligned}
$$

So, for instance, $n=0$ corresponds to (2.22), while $n=12$ corresponds to (2.20)-(2.21). It is amusing to note that the "half-reduced" transform with $n=6$ gives the simplest result, essentially $\left|Z_{\text {top }}\right|^{2}$ with no volume factor.

\section{3. $\mathcal{N}=4$ Black Holes}

\subsection{Preliminaries}

Having computed the OSV transform of the exact $\mathcal{N}=8$ degeneracies, let us now consider the analogous problem for the exact degeneracies of $1 / 4 \mathrm{BPS} \mathcal{N}=4$ black holes. We will consider type IIA string theory compactified on $K 3 \times T^{2}$. The BPS black holes can then be described as supersymmetric bound states of branes and strings wrapped on various cycles in $K 3 \times T^{2}$. A formula for their exact degeneracy was conjectured in [22] and derived in [20]. It takes the form 0

$\Omega_{\mathcal{N}=4}(p, q) \equiv \sum_{J^{3}, B P S \text { states }}(-)^{2 J^{3}}=d\left(q_{e}^{2}, q_{m}^{2}, q_{e} \cdot q_{m}\right)=\oint d \rho d \sigma d \nu \frac{e^{\pi i\left(\rho q_{m}^{2}+\sigma q_{e}^{2}+(2 \nu-1) q_{e} \cdot q_{m}\right)}}{\Phi(\rho, \sigma, \nu)}$

where the ingredients of the RHS are

1. $\Phi(\rho, \sigma, \nu)$, the unique weight 10 automorphic form of the modular group $S p(2, \mathbb{Z})$. Although quite complicated in general, $\Phi$ simplifies near "rational quadratic divisors" (RQDs), along which $1 / \Phi$ has double poles. For details about the approximation of $\Phi$ by rational quadratic divisors, see [22] and also section 3 of [6]. The divisor whose contribution dominates the asymptotic degeneracy is

$$
\rho \sigma+\nu-\nu^{2}=0
$$

5 Note the extra factor of $(-1)^{q_{e} \cdot q_{m}}$ relative to [22]. This factor was missed previously, and it is a consequence of the $4 \mathrm{D}-5 \mathrm{D}$ correspondence [24,20]. We will see below that this factor is essential in order to obtain the correct OSV transform. 
The contributions of the other divisors are suppressed by $\mathcal{O}\left(e^{-Q^{2}}\right)$, which is expected to be non-perturbative from the point of view of topological strings. (In appendix A, we study the effect of these subleading divisors and verify that they are indeed nonperturbative.) Working perturbatively in $1 / Q$, we can make the approximation

$$
\frac{1}{\Phi(\rho, \sigma, \nu)}=\frac{\sigma^{12}}{\left(\rho \sigma+\nu-\nu^{2}\right)^{2}} \eta\left(\frac{\rho \sigma-\nu^{2}}{\sigma}\right)^{-24} \eta\left(-\frac{\rho}{\rho \sigma-\nu^{2}}\right)^{-24}+\mathcal{O}\left(\left(\rho \sigma+\nu-\nu^{2}\right)^{0}\right)
$$

2. $q_{e}^{2}, q_{m}^{2}$ and $q_{e} \cdot q_{m}$, invariants of the $S O(6,22 ; \mathbb{Z})$ subgroup of the full $\mathcal{N}=4$ U-duality group $S L(2, \mathbb{Z}) \times S O(6,22 ; \mathbb{Z})$. These invariants encode the charges of various branes and strings wrapped on the cycles of $K 3 \times T^{2}$. Explicitly, we have

$$
\begin{aligned}
& q_{e}^{2}=2 q_{0} p^{1}+C^{M N} q_{M} q_{N} \\
& q_{m}^{2}=2 p^{0} q_{1}+C_{M N} p^{M} p^{N} \\
& q_{e} \cdot q_{m}=p^{0} q_{0}+p^{1} q_{1}-p^{M} q_{N}
\end{aligned}
$$

where $M, N=2, \ldots, 27$, and

$$
C_{M N} x^{M} y^{N}=C_{a b} x^{a} y^{b}+x^{24} y^{27}+x^{25} y^{26}+x^{26} y^{25}+x^{27} y^{24}
$$

with $C_{a b}, a=2, \ldots, 23$ the intersection form on $H^{2}(K 3)$. Here the electric charges are given by the following: $q_{0}$ is D0-charge; $q_{1}$ is $T^{2}$-wrapped D2 charge; $q_{a}, a=2, \ldots 23$ is $K 3$-wrapped D2 charge; and $q_{i}, i=24, \ldots 27$ are momentum and winding modes of $K 3 \times S^{1}$-wrapped NS5 branes. Meanwhile, the magnetic charges correspond to the following: $p^{0}$ is D6-charge; $p^{1}$ is $K 3$-wrapped D4 charge; $p^{a}$ are $T^{2} \times(\mathrm{K} 3$ cycle)wrapped D4 charge; and $p^{i}$ are F-string $T^{2}$ momentum/winding modes.

\subsection{The full OSV transform}

Just as in the $\mathcal{N}=8$ case, we shall restrict to the case $p^{0}=0$ which greatly simplifies the calculations. We will use the shorthand notation

$$
Q=q_{e}^{2}, \quad P=q_{m}^{2}, \quad R=q_{e} \cdot q_{m}
$$

The black hole partition sum can be written

$$
\begin{aligned}
Z_{\mathcal{N}=4}(p, \phi)= & \sum_{q_{0}, q_{1}, q_{M}} d(Q, P, R) e^{-q_{0} \phi^{0}-q_{1} \phi^{1}-q_{M} \phi^{M}} \\
= & \left(p^{1}\right)^{-2} \sum_{q_{M}} \sum_{\phi^{0,1} \rightarrow \phi^{0,1}+2 \pi i k^{0,1}} \sum_{Q, R} d(Q, P, R) \\
& \quad \times \exp \left[-\frac{\phi^{0}}{2 p^{1}}\left(Q-C^{M N} q_{M} q_{N}\right)-\frac{\phi^{1}}{p^{1}}\left(R+p^{M} q_{M}\right)-q_{M} \phi^{M}\right]
\end{aligned}
$$


where $k^{0,1}$ are summed in the range $0, \cdots, p^{1}-1$ (note that $Q$ is even). The sum over $Q$ and $R$ yields (by definition)

$$
\sum_{Q, R} d(Q, P, R) e^{-\frac{\phi^{0}}{2 p^{1}} Q-\frac{\phi^{1}}{p^{1}} R}=\oint d \rho \frac{e^{\pi i \rho P}}{\Phi\left(\rho, \sigma=\frac{\phi^{0}}{2 \pi i p^{1}}, \nu=\frac{\phi^{1}}{2 \pi i p^{1}}+\frac{1}{2}\right)}
$$

The idea is to compute the contour integral in (3.8) in terms of the residues at the relevant RQDs. As discussed above, the dominant contribution to the integral is expected to be given by the RQD (3.2)-(3.3). Integrating $\rho$ around this RQD yields

$$
\begin{aligned}
\oint d \rho \frac{e^{\pi i \rho P}}{\Phi\left(\rho, \sigma_{*}, \nu_{*}\right)} & =\sigma^{10} \partial_{\rho}\left[\frac{e^{\pi i \rho P}}{\eta\left(\frac{\rho \sigma-\nu^{2}}{\sigma}\right)^{24} \eta\left(-\frac{\rho}{\rho \sigma-\nu^{2}}\right)^{24}}\right]_{\rho=\rho_{*}, \sigma=\sigma_{*}, \nu=\nu_{*}}+\ldots \\
& =\frac{\sigma_{*}^{10} e^{\pi i \rho_{*} P}}{\eta\left(-\frac{\nu_{*}}{\sigma_{*}}\right)^{24} \eta\left(\frac{\rho_{*}}{\nu_{*}}\right)^{24}}\left[\pi i P-24 \frac{\eta^{\prime}\left(-\frac{\nu_{*}}{\sigma_{*}}\right)}{\eta\left(-\frac{\nu_{*}}{\sigma_{*}}\right)}-24 \frac{\eta^{\prime}\left(\frac{\rho_{*}}{\nu_{*}}\right)}{\eta\left(\frac{\rho_{*}}{\nu_{*}}\right)}\right]+\ldots
\end{aligned}
$$

where we have defined

$$
\sigma_{*}=\frac{\phi^{0}}{2 \pi i p^{1}}, \quad \nu_{*}=\frac{\phi^{1}}{2 \pi i p^{1}}+\frac{1}{2}, \quad \rho_{*}=\frac{\nu_{*}^{2}-\nu_{*}}{\sigma_{*}}=\frac{\left(\pi p^{1}\right)^{2}+\left(\phi^{1}\right)^{2}}{2 \pi i p^{1} \phi^{0}},
$$

and ... refers to the contributions of the other RQDs. In appendix A, we study these contributions, and we show that they are indeed nonperturbative in $g_{\text {top }}$ relative to the dominant contribution (3.9).

This leaves the sum over $q_{M}$, which can be evaluated using a (formal) Poisson resummation (as for $\mathcal{N}=8$, there are issues of convergence we are glossing over, since $C^{M N}$ is not positive-definite):

$$
\sum_{q_{M}} e^{\frac{\phi^{0}}{2 p^{1}} C^{M N} q_{M} q_{N}-\left(\frac{\phi^{1}}{p^{1}} p^{M}+\phi^{M}\right) q_{M}}=\left(\frac{p^{1}}{\phi^{0}}\right)^{13} \sum_{\phi^{M} \rightarrow \phi^{M}+2 \pi i k^{M}} e^{-\frac{C_{M N}\left(p^{1} \phi^{M}+\phi^{1} p^{M}\right)\left(p^{1} \phi^{N}+\phi^{1} p^{N}\right)}{2 p^{1} \phi^{0}}}
$$

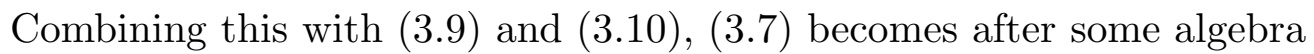

$$
\begin{aligned}
Z_{\mathcal{N}=4}=\sum_{\phi \rightarrow \phi+2 \pi i k} \frac{p^{1}}{\left(\phi^{0}\right)^{3}} & \frac{\exp \left(\frac{C_{M N}\left(\pi^{2} p^{1} p^{M} p^{N}-2 \phi^{1} p^{M} \phi^{N}-p^{1} \phi^{M} \phi^{N}\right)}{2 \phi^{0}}\right)}{\eta\left(\frac{\pi p^{1}-i \phi^{1}}{i \phi^{0}}\right)^{24} \eta\left(\frac{\pi p^{1}+i \phi^{1}}{i \phi^{0}}\right)^{24}} \\
& \times\left[\pi i C_{M N} p^{M} p^{N}-24 \frac{\eta^{\prime}\left(\frac{\pi p^{1}-i \phi^{1}}{i \phi^{0}}\right)}{\eta\left(\frac{\pi p^{1}-i \phi^{1}}{i \phi^{0}}\right)}-24 \frac{\eta^{\prime}\left(\frac{\pi p^{1}+i \phi^{1}}{i \phi^{0}}\right)}{\eta\left(\frac{\pi p^{1}+i \phi^{1}}{i \phi^{0}}\right)}\right]+\ldots
\end{aligned}
$$

In the next subsection, we will see how this rather complicated result of the OSV transform has an extremely simple interpretation in terms of the topological string on $K 3 \times T^{2}$. 


\subsection{Rewriting in terms of the topological string on $K 3 \times T^{2}$}

The topological string on $K 3 \times T^{2}$ is only slightly less trivial than the topological string on $T^{6}$. The prepotential consists of a tree-level term together with a one-loop correction coming from worldsheet instantons:

$$
Z_{\text {top }}=e^{F_{\text {top }}}, \quad F_{\text {top }}=\frac{(2 \pi i)^{3} C_{M N} t^{M} t^{N} t^{1}}{2 g_{\text {top }}^{2}}-24 \log \eta\left(t^{1}\right)
$$

Here $t^{1}, t^{M}, t^{N}, M, N=2, \ldots, 27$ are the Kähler moduli of $K 3 \times T^{2}$. In particular, $t^{1}$ corresponds to the complexified Kähler modulus of $T^{2}$. Just as for $\mathcal{N}=8$, we have generalized the notion of $F_{\text {top }}$ to include the 4 moduli in the $\mathcal{N}=2$ gravitini multiplets, $t^{24, \ldots, 27}$. Again, this is well-motivated from U-duality, and it correctly reproduces the result of the OSV transform.

The attractor equations work exactly the same as for $\mathcal{N}=8$ (2.15). Explicitly, we have (recall we are setting $p^{0}=0$ )

$$
t^{M}=\frac{\pi p^{M}+i \phi^{M}}{i \phi^{0}}, \quad t^{1}=\frac{\pi p^{1}+i \phi^{1}}{i \phi^{0}} \quad g_{t o p}=\frac{4 \pi^{2}}{\phi^{0}}
$$

Finally, we find the Kähler potential in this case to be:

$$
e^{-K}=-\frac{2 i p^{1}}{\phi^{0}}\left[\pi i C_{M N} p^{M} p^{N}-\frac{24 \eta^{\prime}\left(\frac{\pi p^{1}-i \phi^{1}}{i \phi^{0}}\right)}{\eta\left(\frac{\pi p^{1}-i \phi^{1}}{i \phi^{0}}\right)}-\frac{24 \eta^{\prime}\left(\frac{\pi p^{1}+i \phi^{1}}{i \phi^{0}}\right)}{\eta\left(\frac{\pi p^{1}+i \phi^{1}}{i \phi^{0}}\right)}\right]
$$

Notice that we have generalized slightly the definition of the Kähler potential to include the one-loop topological string amplitude (the second and third terms of (3.15), due to world sheet instantons). Normally, $e^{-K}$ is computed using the tree-level prepotential alone. Thus, we can think of $e^{-K}$ defined in (3.15) as a "quantum-corrected" Kähler potential.

Now we have all the ingredients necessary to interpret the OSV transform (3.12) in terms of the topological string on $K 3 \times T^{2}$. In fact, using (3.13)-(3.15), the complicated result (3.12) simplifies considerably:

$$
Z_{\mathcal{N}=4}=\sum_{\phi \rightarrow \phi+2 \pi i k}\left|Z_{\text {top }}\right|^{2}\left|g_{\text {top }}\right|^{2} e^{-K}+\ldots
$$

Again, keep in mind that we have made the crucial restriction $p^{0}=0$ in deriving (3.16). We also recall from (3.7) that the sum over $\phi^{0}$ and $\phi^{1}$ shifts only runs over the range $0 \leq k^{0}, k^{1}<p^{1}$. 
Once again, we can rewrite the answer in terms of the (quantum-corrected) volume of $V_{K 3 \times T^{2}}$ :

$$
V_{K 3 \times T^{2}}=\left|g_{t o p}\right|^{2} e^{-K}
$$

Then (3.16) becomes even simpler,

$$
Z_{\mathcal{N}=4}=\sum_{\phi \rightarrow \phi+2 \pi i k}\left|Z_{t o p}\right|^{2} V_{K 3 \times T^{2}}+\ldots
$$

This should be compared with the analogous $\mathcal{N}=8$ result (2.21). Aside from the factors of $g_{t o p}$ appearing in the latter, which could perhaps be absorbed into the definition of $Z_{t o p}$, we see that the two results are in complete agreement.

\subsection{The general OSV transform}

Once again we can transform the black hole degeneracy only with respect to only $q_{0}$ and the charges $q_{a=1, \ldots, 23}$ which are associated to $\mathcal{N}=2$ vector multiplets. The calculation is nearly identical to the full transform discussed above; the only difference is the power of $p^{1} / \phi^{0}$ in (3.11) becomes 11 instead of 13 . The resulting "reduced" BH partition function is

$$
Z_{\mathcal{N}=4}^{r e d}=\sum_{\phi \rightarrow \phi+2 \pi i k}\left|Z_{t o p}\right|^{2} \frac{V_{K 3}}{V_{T^{2}}}+\cdots
$$

where we have defined $V_{T^{2}}=\left|\operatorname{Im} t^{1}\right|=\frac{\pi p^{1}}{\phi^{0}}$ and $V_{K 3}=\frac{V_{K 3 \times T^{2}}}{V_{T^{2}}}$.

Finally, let us also consider the most general "reduced" partition function obtained by summing up $0 \leq n \leq 4$ gravitini charges. The result is:

$$
Z_{\mathcal{N}=4}^{(n)}=\sum_{\phi \rightarrow \phi+2 \pi i k}\left|Z_{t o p}\right|^{2} V_{K 3}\left(V_{T^{2}}\right)^{n / 2-1}+\cdots
$$

\section{Summary and Discussion}

\subsection{Summary of results}

In the previous sections we have evaluated the OSV transform for $1 / 4$ and 1/8 BPS black holes in type IIA compactification on $K 3 \times T^{2}$ and $T^{6}$, respectively. Apart from the requirement of vanishing D6 brane charge $\left(p^{0}=0\right)$, the black holes we considered had completely general charge configurations. In particular, they could either include the charges in $\mathcal{N}=2$ gravitini multiplets, or not. 
In all cases, we found that the results take the form

$$
Z_{B H}^{(n)}(p, \phi) \equiv \sum_{q} \Omega_{B H}(p, q) e^{-q \phi}=\sum_{k \in \Gamma} G^{(n)}(p, \phi+2 \pi i k)
$$

with

$$
G^{(n)}(p, \phi)=\left|Z_{t o p}\right|^{2} \times(\text { simple factor })
$$

where $n$ denotes the number of gravitini charges being summed over $(n=4$ and $n=12$ for the full $\mathcal{N}=4$ and $\mathcal{N}=8$ transforms, respectively), and the precise form of the simple factor depends on the transform and degeneracy being considered.

In more detail, we found that up to nonperturbative corrections in $g_{t o p}$, the answer for $G$ was

$$
\begin{aligned}
& G_{\mathcal{N}=8}^{(n)}(p, \phi)=\left|Z_{\text {top }}\right|^{2}\left|g_{\text {top }}\right|^{8}\left(V_{T^{6}}\right)^{n / 6-1} \\
& G_{\mathcal{N}=4}^{(n)}(p, \phi)=\left|Z_{\text {top }}\right|^{2} V_{K 3}\left(V_{T^{2}}\right)^{n / 2-1}
\end{aligned}
$$

In addition, we were able to sum up the nonperturbative corrections to the $\mathcal{N}=8$ answer, yielding an exact result in this case:

$$
G_{\mathcal{N}=8}^{(n)}(p, \phi)=\left|Z_{\text {top }}\right|^{2}\left|g_{\text {top }}\right|^{8}\left(V_{T^{6}}\right)^{n / 6-1} \tilde{F}\left(\frac{i V_{T^{6}}}{\left|g_{\text {top }}\right|^{2}}\right)
$$

with the modular form $\tilde{F}(\rho)$ given by $(2.12)$.

Despite the discrepancies from $\left|Z_{\text {top }}\right|^{2}$, the OSV transforms of the $\mathcal{N}=4$ and $\mathcal{N}=8$ degeneracies have very similar structures. Clearly, there are patterns here that need to be better understood!

\subsection{The holomorphic anomaly and the metric on moduli space}

Let us now examine the most general OSV transforms (2.23) and (3.20) in more detail. We can write them in terms of a natural metric on the space of $X^{\Lambda}$ 's - the one that governs the kinetic term of the corresponding vector fields in supergravity:

$$
g_{\Lambda \Sigma}^{(c l)} \equiv \partial_{\Lambda} \partial_{\bar{\Sigma}} e^{-K^{(c l)}}=\frac{1}{\pi} \operatorname{Re} \partial_{\Lambda} \partial_{\Sigma} F_{t o p}^{(c l)}
$$

where the $(c l)$ superscript denotes the classical tree-level contribution; and $\Lambda, \Sigma=$ $0, \ldots, 23+n$ for $K 3 \times T^{2}$ and $\Lambda, \Sigma=0, \ldots, 15+n$ for $T^{6}$. A short calculation using 
the appropriate $F_{t o p}^{(c l)}$ shows that the determinant of $g^{(c l)}$ takes the form (disregarding overall numerical factors)

$$
\begin{array}{lc}
\operatorname{det} g^{(c l)}=\left(\operatorname{Im} t^{1}\right)^{20+n}\left(V_{K 3 \times T^{2}}^{(c l)}\right)^{2} & \left(K 3 \times T^{2}\right) \\
\operatorname{det} g^{(c l)}=\left(V_{T^{6}}\right)^{6+n / 3} & \left(T^{6}\right)
\end{array}
$$

Therefore, the general OSV transforms (2.23) and (3.20) can be written nicely in terms of this metric as

$$
G^{(n)}(p, \phi)=\left|Z_{\text {top }}^{\prime}\right|^{2} \sqrt{\operatorname{det} g^{(c l)}}\left(\frac{V_{X}}{V_{X}^{(c l)}}\right)
$$

where $X=K 3 \times T^{2}$ or $T^{6}$, and

$$
\left|Z_{\text {top }}^{\prime}\right|^{2}= \begin{cases}\left|Z_{\text {top }}\right|^{2}\left(\operatorname{Im} t^{1}\right)^{-12} & X=K 3 \times T^{2} \\ \left|Z_{\text {top }}\right|^{2} e^{4 K} & X=T^{6}\end{cases}
$$

Notice how the explicit $n$ dependence has been completely absorbed into the determinant of the metric. In particular, $\left|Z_{\text {top }}^{\prime}\right|^{2}$ is independent of $n$. In the case of $K 3 \times T^{2}$, we recognize $\left|Z_{\text {top }}^{\prime}\right|^{2}$ to be the square of the topological string partition function including the holomorphic anomaly [28]!

The factor $e^{4 K}$ in the $\mathcal{N}=8$ case is more mysterious, since the topological string on $T^{6}$ has no holomorphic anomaly. Let us consider the special case when only the Kähler moduli $t^{1}, t^{2}, t^{3}$ are turned on, and then

$$
e^{4 K}=\left|g_{t o p}\right|^{8}\left(\operatorname{Im} t^{1} \operatorname{Im} t^{2} \operatorname{Im} t^{3}\right)^{-4}
$$

Apart from the factor $\left|g_{\text {top }}\right|^{8}$, which also showed up in (4.3), the RHS of (4.9) is identical to the 1-loop holomorphic anomaly in toroidal orbifold models, such as $T^{6} / \mathbb{Z}_{3} \times \mathbb{Z}_{3}$. We suspect that (4.9) may be interpreted as the holomorphic anomaly of a modified genus 1 amplitude defined by a new index. It would be interesting to understand this.

Although (4.7) is fairly nice overall, it does contain the rather ugly factor $\frac{V_{X}}{V_{X}^{(c l)}}$. We will attempt to explain this factor as follows. It differs from unity only in the case of $X=K 3 \times T^{2}$, so let us focus on that case. From the form of $\operatorname{det} g^{(c l)}$ in (4.6), it is tempting to propose the existence of a "quantum-corrected" metric on the space of $X^{\Lambda}$ 's, $g^{(q)}$, whose determinant gives the quantum-corrected volume, i.e.

$$
\sqrt{\operatorname{det} g^{(q)}}=\left(\operatorname{Im} t^{1}\right)^{10+n / 2} V_{K 3 \times T^{2}}
$$


Then in terms of this metric, (4.7) reduces to

$$
G^{(n)}(p, \phi)=\left|Z_{\text {top }}^{\prime}\right|^{2} \sqrt{\operatorname{det} g^{(q)}}
$$

and the calculation of the OSV transform (4.1) becomes

$$
Z_{B H}^{(n)}(p, \phi)=\sum_{\phi \rightarrow \phi+2 \pi i k}\left|Z_{t o p}^{\prime}\right|^{2} \sqrt{\operatorname{det} g^{(q)}}
$$

where again, all of the explicit $n$ dependence is contained in the determinant of the metric. Note that (4.11)-(4.12) also apply to $X=T^{6}$, since in that case it is natural to suppose that $g^{(q)}=g^{(c l)}$.

Introducing a quantum-corrected metric $g^{(q)}$ may seem ad hoc, but evidence for its existence comes from the following observation. If we suppose that the quantum corrections to the metric are somehow the result of the one-loop worldsheet instanton corrections in (3.13), then it is natural to assume that

$$
\delta g_{\Lambda \Sigma}=g_{\Lambda \Sigma}^{(q)}-g_{\Lambda \Sigma}^{(c l)}
$$

only has nonzero components for $\Lambda, \Sigma=0,1$ and is a function only of $X^{0}$ and $X^{1}$. Imposing that $\delta g$ be real, we find that the nonzero components of $\delta g^{(q)}$ are given uniquely by

$$
\left(\begin{array}{ll}
\delta g_{00} & \delta g_{01} \\
\delta g_{10} & \delta g_{11}
\end{array}\right)=\frac{24\left|g_{t o p}\right|^{2}}{\operatorname{Im} t^{1}} \operatorname{Re}\left(\frac{\eta^{\prime}\left(t^{1}\right)}{\eta\left(t^{1}\right)}\right)\left(\begin{array}{cc}
\left|t^{1}\right|^{2} & -\operatorname{Re} t^{1} \\
-\operatorname{Re} t^{1} & 1
\end{array}\right)
$$

The uniqueness and the simplicity of (4.14) strongly suggests that the idea of a quantumcorrected metric on the space of $X^{\Lambda}$ 's should be taken seriously.

To summarize, eq. (4.11)-(4.12) provide a unified description of the most general OSV transform, for both $\mathcal{N}=4$ and $\mathcal{N}=8$ supersymmetry, in terms of the topological string amplitude with holomorphic anomaly and the quantum metric on the moduli space. The latter provides a natural measure for the wavefunction interpretation of $Z_{\text {top }}$. A similar measure factor was proposed in [5] (see e.g. eq. (6.6) of that paper); however, we note that our answer differs significantly from that of [5]. 


\subsection{D-instantons}

As discussed in [15] and the introduction, the sum over shifts $\phi \rightarrow \phi+2 \pi i k$ in (4.1) is entirely expected.6 After all, the OSV transform involves a sum of $e^{-q \cdot \phi}$ over integral charges $q$; therefore the result must be periodic in imaginary $\phi$. However, the interpretation of the sum from the topological string point of view is intriguing.

First, restricting to the case $p^{0}=0$, we have $\phi^{0}=\frac{4 \pi^{2}}{g_{t o p}}$. Thus, the periodicity in imaginary $\phi^{0}$ is reminiscent of the periodicity in the theta angle in Yang-Mills theory. The latter periodicity signals the quantization of instanton charges, and so one might expect the periodicity in imaginary $\phi^{0}$ to be related to the quantization of D-instanton charges. In fact, the OSV transform

$$
\sum_{q} \Omega(p, q) e^{-q \cdot \phi}=\sum_{q} \Omega(p, q)(-)^{p \cdot q} \exp \left[-\frac{4 \pi^{2}}{g_{t o p}}\left(q_{0}+q_{A} t^{A}\right)\right]
$$

can be interpreted as an sum over D-instanton contributions in the topological B-model. Our result can then be thought of as a relation between the partition function of Bbranes and the perturbative partition function of the A-model. This is reminiscent of the topological S-duality [29] although the details seem very different.

Meanwhile, the sum over shifts in $\phi^{A}$ can be written as shifts in the Kähler moduli

$$
t^{A} \rightarrow t^{A}+\frac{1}{2 \pi i} k^{A} g_{t o p}
$$

is entirely analogous to the sum in the 2DYM/topological string correspondence [2],

$$
t \rightarrow t+m \lg _{t o p}, \quad \bar{t} \rightarrow \bar{t}-m l g_{t o p}
$$

(The apparent discrepancy of $2 \pi i$ is because $t=2 \pi i X^{1} / X^{0}$ in [2].) This was interpreted in [2] as a summation over contribution from sectors with RR 2-form fluxes. It would be nice to understand the analogous statement in our context.

\footnotetext{
${ }^{6}$ Of course, it is still nontrivial that the summand is essentially $\left|Z_{t o p}\right|^{2}$ times a measure factor.
} 


\subsection{Non-perturbative corrections}

The full result of the OSV transform for the $\mathcal{N}=8$ black hole $(2.20)$ can be thought of as the non-perturbative completion of the topological string amplitude on $T^{6}$. Let us briefly discuss the nature of the non-perturbative corrections. They are suppressed by exponential factors of the form

$$
\exp \left(-e^{-K}\right)=\exp \left(-V_{T^{6}} / g_{\text {top }}^{2}\right)
$$

Note that the exponent is proportional to $1 / g_{\text {top }}^{2}$ rather than $1 / g_{t o p}$, which is reminiscent of the instanton corrections in a gauge theory rather than D-instanton effects in string theory.

The nonperturbative corrections in the OSV transform for the $\mathcal{N}=4$ black hole is discussed in Appendix A. We find that again they behave as $\exp \left(-\alpha V / g_{\text {top }}^{2}\right)$, although unlike the $\mathcal{N}=8$ case, the exponent $\alpha$ is bounded both from above and from below.

It would be interesting to understand the physical meaning of these corrections, and in particular the possible connection to the baby universe interpretation as in [11]. We should note that the $\mathcal{N}=8$ black hole degeneracy was derived in [21] while ignoring the effects due to fragmentation. Such effects might need to be taken into account in order to accurately match the nonperturbative corrections with multi-black hole states and the corresponding interpretation in the topological string.

\section{Acknowledgments:}

We thank G. Moore, A. Neitzke, V. Pestun, and A. Strominger for useful discussions. The research of DS is supported in part by an NSF Graduate Research Fellowship and by NSF grant PHY-0243680. The research of XY is supported in part by DOE grant DEFG02-91ER40654. Any opinions, findings, and conclusions or recommendations expressed in this material are those of the author(s) and do not necessarily reflect the views of the National Science Foundation.

\section{Appendix A. Nonperturbative Corrections to the $\mathcal{N}=4$ OSV Transform}

In this appendix, we will analyze the corrections to the $\mathcal{N}=4$ OSV transform, the $\ldots$ in section 3 . We will show that these corrections are nonperturbative in $g_{\text {top }}$, justifying our neglect of them in the text. 
As discussed in section 3, these corrections come from the residues of rational quadratic divisors other than (3.2). Following the appendix of [22], the most general RQD is characterized by five integers $\mathbf{v}=(k, l, m, a, c)$ satisfying one constraint

$$
4 a c-4 k l+m^{2}-1=0
$$

The RQD is then

$$
a\left(\rho \sigma-\nu^{2}\right)+k \rho+l \sigma+m \nu+c=0
$$

Substituting $\sigma=\frac{\phi^{0}}{2 \pi i p^{1}}, \nu=\frac{\phi^{1}}{2 \pi i p^{1}}+\frac{1}{2}$, we can solve for $\rho$ :

$$
\rho(\mathbf{v})=-\frac{l}{a}+\frac{\left((a-m) \pi p^{1}+i a \phi^{1}\right)^{2}-\left(\pi p^{1}\right)^{2}}{2 a \pi p^{1}\left(2 k \pi p^{1}-i a \phi^{0}\right)}
$$

The RQD (3.2) corresponds to $a=m=1, k=l=c=0$, in which case (A.3) reduces to the third equation of $(3.10)$.

Repeating the calculation in section 3.1, we see that the general RQD contributes an amount to $Z_{\mathcal{N}=4}(p, \phi)$ which goes like

$$
\delta Z_{\mathcal{N}=4} \sim e^{\pi i \rho(\mathbf{v}) C_{M N} p^{M} p^{N}}
$$

Let us now compare this with the contribution from (3.2), which goes like $e^{\pi i \rho_{*} C_{M N} p^{M} p^{N}}$. We have

$$
\frac{\delta Z_{\mathcal{N}=4}}{Z_{\mathcal{N}=4}}=e^{\pi i\left(\rho(\mathbf{v})-\rho_{*}\right) C_{M N} p^{M} p^{N}}
$$

The real part of this exponent is

$$
\begin{aligned}
\delta F & \equiv \operatorname{Re}\left[\pi i\left(\rho(\mathbf{v})-\rho_{*}\right) C_{M N} p^{M} p^{N}\right] \\
& =-\frac{\pi^{2} C_{M N} p^{M} p^{N} p^{1}}{2 \phi^{0}}\left(\frac{\left((a-m) \phi^{0}+2 k \phi^{1}\right)^{2}+\left(2 \pi k p^{1}\right)^{2}+\left(a^{2}-1\right)\left(\phi^{0}\right)^{2}}{\left(2 \pi k p^{1}\right)^{2}+\left(a \phi^{0}\right)^{2}}\right)
\end{aligned}
$$

This quantity is clearly negative, provided we assume that $\frac{C_{M N} p^{M} p^{N} p^{1}}{\phi^{0}}>0$ and take $a \geq 1$. The condition $a \geq 1$ follows from the contour of $\rho$ integration. The contour must necessarily avoid the $a=0$ RQDs, since otherwise the $a=0$ RQDs would dominate the exact degeneracies and ruin the large charge asymptotics $\Omega_{\mathcal{N}=4}(p, q) \sim e^{S_{c l}(p, q)}$.

In fact, we are free to choose the contour such that only a single $a=1 \mathrm{RQD}$, say $k=l=c=0$ and $m=1$, contributes. The reason is that the other $a=1$ RQDs are related to this one by integer shifts of $\rho, \nu, \sigma$, and so they all contribute identically to 
$Z_{\mathcal{N}=4}(p, \phi)$. This is nontrivial from the point of view of the above expressions; however, it is easy to see if we write $Z_{\mathcal{N}=4}(p, \phi)$ as

$$
Z_{\mathcal{N}=4}=\frac{1}{\left(p^{1}\right)^{2}} \sum_{k^{0}=0}^{p^{1}-1} \sum_{k^{1}=0}^{p^{1}-1} Z_{\mathcal{N}=4}^{\prime}\left(p, \phi^{0}+2 \pi i k^{0}, \phi^{1}+2 \pi i k^{1}, \phi^{M}\right)
$$

with

$Z_{\mathcal{N}=4}^{\prime}(p, \phi) \equiv \sum_{q_{M}} \oint \frac{d \rho}{\Phi\left(\rho, \sigma=\frac{\phi^{0}}{2 \pi i p^{1}}, \nu=\frac{\phi^{1}}{2 \pi i p^{1}}+\frac{1}{2}\right)} e^{\pi i \rho C_{M N} p^{M} p^{N}+\frac{\phi^{0}}{2 p^{1}} C^{M N} q_{M} q_{N}+\frac{\phi^{1}}{p^{1}} p^{M} q_{M}-\phi^{M} q_{M}}$

The $(a=1, k, l, m, c)$ RQD with $\sigma=\frac{\phi^{0}}{2 \pi i p^{1}}$ and $\nu=\frac{\phi^{1}}{2 \pi i p^{1}}+\frac{1}{2}$ can be brought to the form $(a=1,0,0,1,0)$ by shifting $\phi^{1}$ and $\phi^{0}$ by $2 \pi i p^{1} s$ and $2 \pi i p^{1} t$ for some integers $s$ and $t$. Since $Z_{\mathcal{N}=4}^{\prime}$ is clearly invariant under such shifts, this shows that all the $a=1$ RQDs contribute identically to $Z_{\mathcal{N}=4}$. By choosing the contour so that only one $a=1$ RQD contributes, we avoid overcounting.

Therefore, we have shown that aside from the contribution of the $a=1$ RQD (3.2), the only other contributions to the black hole partition function are from RQDs with $a>1$, and all of these are nonperturbative. Let us now examine the $a>1$ RQDs in more detail.

Although we have considered the most general RQD, we do not expect all of them to contribute to the exact degeneracies, for the following reason. $\Phi$ is only holomorphic in the Siegel upper half plane, defined by

$$
\|\Omega\| \equiv \operatorname{det} \operatorname{Im}\left(\begin{array}{ll}
\rho & \nu \\
\nu & \sigma
\end{array}\right)>0
$$

Therefore, we should only consider the contributions of RQDs which satisfy (A.9). Substituting once again $\sigma=\frac{\phi^{0}}{2 \pi i p^{1}}, \nu=\frac{\phi^{1}}{2 \pi i p^{1}}+\frac{1}{2}$, and $\rho=\rho(\mathbf{v})$, we find a simple relation between $\delta F$ and $\|\Omega\|$ :

$$
\delta F=\frac{2 \pi^{2} C_{M N} p^{M} p^{N} p^{1}}{\phi^{0}}\left(\|\Omega\|-\frac{1}{4}\right)
$$

Therefore, the exponential suppression (a negative quantity) of the $a>1$ RQDs is bounded from below by

$$
\delta F>-\frac{1}{4} \frac{2 \pi^{2} C_{M N} p^{M} p^{N} p^{1}}{\phi^{0}}
$$

In addition, one can easily deduce from (A.6) an upper bound on $\delta F$ for $a>1$. Combining these, we conclude that the exponential suppression of the subleading RQDs is contained within a tight band:

$$
-\frac{\pi^{2} C_{M N} p^{M} p^{N} p^{1}}{2 \phi^{0}}<\delta F<-\frac{3}{4} \times \frac{\pi^{2} C_{M N} p^{M} p^{N} p^{1}}{2 \phi^{0}}
$$


The lower bound on $\delta F$ is intriguing. It should be contrasted with the very different behavior of the nonperturbative corrections in the $\mathcal{N}=8$ case (2.20), where we found a series of successively smaller nonperturbative corrections. 


\section{References}

[1] H. Ooguri, A. Strominger and C. Vafa, "Black hole attractors and the topological string," Phys. Rev. D 70, 106007 (2004) arXiv:hep-th/0405146.

[2] C. Vafa, "Two dimensional Yang-Mills, black holes and topological strings," arXiv:hepth/0406058.

[3] A. Dabholkar, "Exact counting of black hole microstates," arXiv:hep-th/0409148.

[4] M. Aganagic, H. Ooguri, N. Saulina and C. Vafa, "Black holes, q-deformed 2d YangMills, and non-perturbative topological strings," Nucl. Phys. B 715, 304 (2005) arXiv:hep-th/0411280.

[5] E. Verlinde, "Attractors and the holomorphic anomaly," arXiv:hep-th/0412139;

[6] G. L. Cardoso, B. de Wit, J. Kappeli and T. Mohaupt, "Asymptotic degeneracy of dyonic $\mathrm{N}=4$ string states and black hole entropy," JHEP 0412, 075 (2004) arXiv:hepth/0412287.

[7] A. Sen, "Black holes, elementary strings and holomorphic anomaly," arXiv:hepth/0502126.

[8] A. Dabholkar, F. Denef, G. W. Moore and B. Pioline, "Exact and asymptotic degeneracies of small black holes," arXiv:hep-th/0502157.

[9] A. Sen, "Black holes and the spectrum of half-BPS states in $N=4$ supersymmetric string theory," arXiv:hep-th/0504005.

[10] M. Aganagic, A. Neitzke and C. Vafa, "BPS microstates and the open topological string wave function," arXiv:hep-th/0504054.

[11] R. Dijkgraaf, R. Gopakumar, H. Ooguri and C. Vafa, "Baby universes in string theory," arXiv:hep-th/0504221.

[12] A. Sen, "Stretching the horizon of a higher dimensional small black hole," arXiv:hepth/0505122.

[13] A. Sen, "Black hole entropy function and the attractor mechanism in higher derivative gravity," arXiv:hep-th/0506177.

[14] B. Pioline, "BPS black hole degeneracies and minimal automorphic representations," arXiv:hep-th/0506228.

[15] A. Dabholkar, F. Denef, G. W. Moore and B. Pioline, "Precision counting of small black holes," arXiv:hep-th/0507014.

[16] J. M. Maldacena, A. Strominger and E. Witten, "Black hole entropy in M-theory," JHEP 9712, 002 (1997) arXiv:hep-th/9711053.

[17] G. Lopes Cardoso, B. de Wit and T. Mohaupt, "Corrections to macroscopic supersymmetric black-hole entropy," Phys. Lett. B 451, 309 (1999) [arXiv:hep-th/9812082.

[18] A. Dabholkar and J. A. Harvey, "Nonrenormalization Of The Superstring Tension," Phys. Rev. Lett. 63, 478 (1989). 
[19] A. Dabholkar, G. W. Gibbons, J. A. Harvey and F. Ruiz Ruiz, "Superstrings And Solitons," Nucl. Phys. B 340, 33 (1990).

[20] D. Shih, A. Strominger and X. Yin, "Recounting dyons in N $=4$ string theory," arXiv:hep-th/0505094.

[21] D. Shih, A. Strominger and X. Yin, "Counting dyons in N = 8 string theory," arXiv:hep-th/0506151.

[22] R. Dijkgraaf, E. Verlinde and H. Verlinde, "Counting dyons in $\mathrm{N}=4$ string theory," Nucl. Phys. B 484, 543 (1997) arXiv:hep-th/9607026.

[23] S. Ferrara and R. Kallosh, "Universality of Supersymmetric Attractors," Phys. Rev. D 54, 1525 (1996) arXiv:hep-th/9603090.

[24] D. Gaiotto, A. Strominger and X. Yin, "New connections between 4D and 5D black holes," arXiv:hep-th/0503217.

[25] E. Cremmer and B. Julia, "The SO(8) Supergravity," Nucl. Phys. B 159, 141 (1979).

[26] V. Balasubramanian, "How to count the states of extremal black holes in $\mathrm{N}=8$ supergravity," arXiv:hep-th/9712215.

[27] R. Kallosh and B. Kol, "E(7) Symmetric Area of the Black Hole Horizon," Phys. Rev. D 53, 5344 (1996) arXiv:hep-th/9602014.

[28] J. A. Harvey and G. W. Moore, "Fivebrane instantons and $\mathrm{R}^{* *} 2$ couplings in $\mathrm{N}=4$ string theory," Phys. Rev. D 57, 2323 (1998) arXiv:hep-th/9610237.

[29] N. Nekrasov, H. Ooguri and C. Vafa, "S-duality and topological strings," JHEP 0410, 009 (2004) arXiv:hep-th/0403167. 